\title{
Coupling Molecular Dynamics and Finite Element Simulations to Investigate the Nearest Neighbor Dependence of Field Evaporation
}

\author{
Travis Withrow ${ }^{1}$, Christian Oberdorfer ${ }^{1}$, Wolfgang Windl ${ }^{1}$ and Emmanuelle A Marquis ${ }^{2}$ \\ 1. The Ohio State University, Department of Materials Science and Engineering, Columbus, OH, USA \\ 2. University of Michigan, Department of Materials Science and Engineering, Ann Arbor, MI, USA
}

Atom probe tomography is an increasingly popular technique for investigating materials problems at the nanoscale, especially with recent advances in the technique. The presence of artifacts in the data generated by the atom probe, however, can make it difficult to draw certain conclusion about the microstructural features of a sample. The origins of these artifacts are still somewhat unclear and therefore can potentially be a hindrance to the usefulness of this technique. Unlike other microscopy techniques, analysis of atom probe data requires detailed knowledge not only about a material's behavior in a strong electric field but also how the evaporation of a material changes based on the specific microstructural characteristics of a material [1].

The origins of these artifacts have been investigated previously by numerical simulations of field evaporation. These electrostatic models have demonstrated a remarkable ability to reproduce and explain a number of artifacts in atom probe data but usually consider a fixed zero barrier field (ZBEF) for each species. Artifacts with subtler and more complicated causes are not treated appropriately by these simplistic models and thus require a more complex simulation framework. The effects of the local neighborhood on the ZBEF are neglected. This is addressed by coupling a state of the art atom probe simulation approach, TAPSim [2], with the well-known molecular dynamics (MD) package, LAMMPS [3]. The inclusion of MD allows for a more complete treatment of field evaporation than has previously been done.

Firstly, these improved simulations are based on a previous study using the image hump model, informed by density functional theory (DFT) results to approximate the local ZBEF. [4] The image-hump model gives an approximation for the ZBEF based on the adhesion energy $(\Lambda)$, the nth ionization energies $\left(I_{i}\right)$, and the work function $(\Phi)[5,6]$ :

$$
Z B E F=4 \pi \varepsilon_{0}(n e)^{-3}\left(\Lambda+\sum_{i=1}^{n} I_{i}+n \phi\right)^{2}
$$

The required parameters are calculated with VASP [7-9] for a number of different adatom configurations - including single adatoms, kink sites, corners and plane edges - to determine inputs for the image hump model. These results allow for a surface dependent ZBEF for the material under study. In simple cases, this behavior can be fitted to a linear nearest neighbor counting model:

$$
Z B E F=F_{0}+\sum_{i=1}^{4} \alpha_{i} n n_{i}
$$

Where $F_{0}$ is the field offset and the $\alpha_{i}$ are the per-atom contributions of the $i^{\text {th }}$ nearest neighbors to the total ZBEF. Figure 1 shows the simulated field evaporation pattern of an fcc [111] orientated emitter sample. The patterns show approximately 80000 evaporation events, equivalent to a few tens of surface layers. In figure 1a, evaporation events are chosen based solely on contribution from the first nearest neighbors $\left(\alpha_{1}\right.$ $\left.\neq 0, \alpha_{i \neq 1}=0\right)$. The pattern is regular and clearly resolved, very similar to what previous simulations have shown. In figure $1 b$, only contributions from the second nearest neighbors are considered $\left(\alpha_{2} \neq 0, \alpha_{i \neq 2}=\right.$ 
0 ), the fine structure of the pattern is lost while other features like poles and major zone lines are still visible. Because in both cases the evaporation still happens atom by atom in a regularly repeating sequence, the depth resolution of the 3D reconstruction does not change. However, the distortion of the pattern caused by including extended neighbor interactions causes a deviation from the ideal evaporation case and decisively decreases the lateral resolution in the reconstruction.

This example illustrates that for an appropriate treatment of these effects, a microscopic model for site dependent evaporation is needed. The addition of LAMMPS to TAPSim allows the derivation of the adhesion energy of every atom on the surface of the sample during the simulation. Computing the atomistic ZBEF allows for the treatment of more complex systems without need to perform computationally expensive DFT calculations and enables the study the evaporation of more complex microstructures. [10]

\section{References:}

[1] EA Marquis and F Vurpillot, Microscopy and Microanalysis 14 (2008), p. 561.

[2] C Oberdorfer, SM Eich and G Schmitz, Ultramicroscopy 128 (2013), p. 55.

[3] S Plimpton, Journal of Computational Physics 117 (1995), p. 1.

[4] L Yao et al Applied Physics Letters 107 (2015), p. 241602.

[5] EW Mueller, Physical Review 102 (1956), p. 618.

[6] RG Forbes, Applied Surface Science 87/88 (1994), p.1.

[7] G Kresse and J Hafner, Physical Review B 47 (1993), p. 558.

[8] G Kresse and J Furthmueller, Computational Materials Science 6 (1996), p. 15.

[9] G Kresse and J Furthmueller, Physical Review B 54 (1996), p. 169.

[10] The authors acknowledge financial support from the Air Force Office of Scientific Research, Award Number FA9550-14-1-0249 and computational support by the Ohio Supercomputer Center, Grant Number PAS0072.

(a)

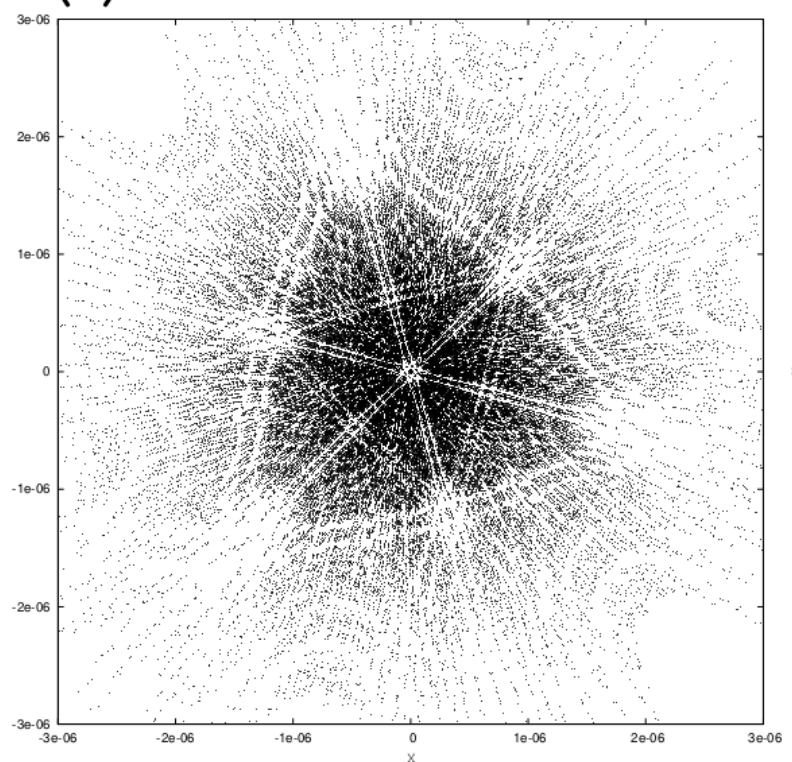

(b)

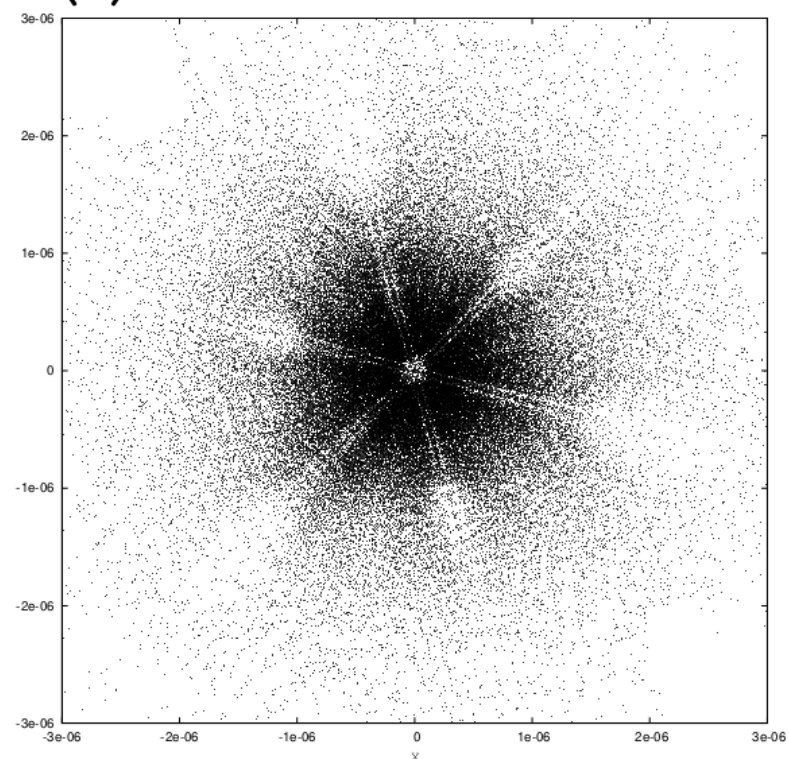

Figure 1. Simulated field evaporation detector patterns of an fcc [111] tip and different parameters in neighbor counting model. a) Only first nearest neighbor interactions. b) Only second nearest neighbor interactions. 